\title{
Measuring Route Diversity in the Internet from Remote Vantage Points
}

\author{
Andrea Di Menna, Tiziana Refice, Luca Cittadini, Giuseppe Di Battista \\ \{dimenna,refice,ratm,gdb\}@dia.uniroma3.it \\ Dipartimento di Informatica e Automazione - Università di Roma Tre
}

\begin{abstract}
Recent works on modeling the Internet topology [8,9] have highlighted how the complexity of relationships between Autonomous Systems (ASes) can not be oversimplified without sacrificing accuracy in capturing route selection. Such a shortcoming can mislead the understanding, hence the prediction, of the BGP (Border Gateway Protocol) behavior. In particular, models that assume an AS to be an atomic entity fail to account for route diversity, informally defined as the selection within a single AS of multiple routes to the same destination prefix. Internet topology models are usually built out of BGP data collected by remote vantage points. Thus, in this paper we aim at extracting and characterizing the route diversity that can be measured using such dataset.

Towards this goal, we devise a methodology to compute route diversity from a continuous stream of collected BGP messages. The analysis of our results shows that (i) accounting for the BGP dynamics allows to extract much more diversity than from a static snapshot of the Internet routing configuration; (ii) route diversity observed for an AS is strongly related to its location in the customer-provider hierarchy; (iii) the distribution of route diversity over ASes is unlikely to be biased by the specific choice of the collection system, while the number of prefixes exhibiting route diversity can depend on both number and location of the vantage points.
\end{abstract}

\section{Introduction}

The topological characteristics of the Internet have been the subject of several research efforts (e.g., $[4,8])$, which significantly helped understand the network behavior. In particular, the Internet can be represented as a graph of administrative domains called Autonomous Systems (ASes), that exchange reachability information using $B G P$ (Border Gateway Protocol) [7], the de-facto standard inter-domain routing protocol.
Recently, [8] proposed a model of interdomain routing which accounts for route diversity, i.e., the simultaneous usage in a single AS of multiple routes towards the same destination prefix. Basically, [8] relaxed the common constraint of ASes being regarded as atomic entities, by identifying in each AS multiple BGP routers which make different decisions about the routing of a specific destination. This model has been exploited to better predict the Internet behavior [8] and to study the interaction between the Internet topology and inter-AS policies [9]. However, the analysis performed in $[8,9]$ only relied on a static snapshot of the network (i.e., a BGP's steady state), while several works (e.g., $[4,10])$ underlined how routing dynamics provide a significant amount of additional information about the Internet topology.

In this paper we propose a technique that takes into account the BGP routing dynamics in order to extract route diversity from BGP data collected by passive remote vantage points. Using our approach, we identify much more diversity than in $[8,9]$. Since route diversity can be exploited to build BGP models that better fit the collected data, we believe that this improvement is relevant, because lots of research projects which aim at studying Internet dynamics rely on public data collected at remote vantage points.

We also provide a characterization of the route diversity as computed by our approach. First, we analyze the relationship between the route diversity observed for an AS and its location in the Internet customer-provider hierarchy. Moreover, we investigate whether the distribution of route diversity over all the ASes is biased by the specific choice of the dataset, in terms of number and location of observation points.

The rest of the paper is organized as follows. Section 2 formally defines the metric we use to model route diversity. In Section 4 we describe our methodology to compute this metric out of BGP update streams. Then (Section 5) we analyze the results obtained by applying our approach to the dataset described in Section 3. 


\section{Modeling the Route Diversity in the Inter- net}

Every BGP router learns through BGP the AS-paths (or routes) it can use to reach any destination prefix on the Internet, selects the one it prefers according to its own configuration, and stores it in its RIB (Routing Information Base). An AS-path (or path) $\pi$ is a sequence of ASes $\pi=\left(a s_{n}, \ldots, a s_{0}\right)$, where $a s_{0}$ is the origin of the prefix. BGP routers establish BGP peering sessions between each other to exchange reachability information by means of $B G P$ updates. Once a BGP router receives from any of its peers an update for a prefix $p$, it recomputes its best path towards $p$, possibly changes its own RIB and propagates the update to its peers. We refer to the case when the new path differs from the old path as (routing) change. In order to better understand the behavior of BGP, special BGP routers (called collector peers or $C P s$ ) have been spread over the Internet by the RIS [1] and RV [2] projects. They act as vantage points and provide historical BGP data.

We define the relationship $X_{A} \neq_{p, t} X_{B}$ to indicate that at time $t$ AS $X$ reaches the prefix $p$ using two different routes, one through AS $A$, the other through AS $B$. Given that every BGP router can use only its current best path to $p$, the simultaneous usage of two distinct paths by AS $X$ implies that $X$ has at least two distinct BGP routers, one $\left(X_{A}\right)$ peering with AS $A$ and the other $\left(X_{B}\right)$ peering with AS $B$. We formalize this intuition through the following property.

Property 1 If there exist two distinct paths $\pi_{1}=$ $(X, A, \ldots)$ and $\pi_{2}=(X, B, \ldots)$ that are both used by $A S X$ to reach prefix $p$ at time $t$, then $X_{A} \neq_{p, t} X_{B}$.

Observe that paths $\pi_{1}$ and $\pi_{2}$ could share a common left subsequence of ASes. In such a scenario, we disambiguate the two (or more) routers that AS $X$ uses to peer with as $A$ by means of numerical superscripts (e.g., $X_{A}^{1} \neq \neq_{p, t} X_{A}^{2}$ ).

The simultaneous usage by a single AS of multiple routes to the same prefix is commonly called route diversity. We distinguish route diversity from path diversity, where the latter refers to the simultaneous availability of multiple routes between the same AS pair.

We extend the definition of $\neq_{p, t}$ to a set of distinct routers $S_{X}=\left\{X_{A}, X_{B}, \ldots\right\}$ (with $\left|S_{X}\right| \geq 2$ ), that we call diversity set. The relation $\neq_{p, t} S_{X}$ means that AS $X$ uses a distinct router to peer with each one of the ASes $\{A, B, \ldots\}$. More formally, $\neq_{p, t} S_{X} \Leftrightarrow \forall\left(X_{A}, X_{B}\right) \in$ $\left(S_{X} \times S_{X}\right), X_{A} \neq_{p, t} X_{B}$. We say that the diversity set $S_{X}$ is associated with AS $X$.

As already pointed out in $[8,9], \neq_{p, t}$ relationships associated with AS $X$ impose a lower bound on the number of BGP routers that $X$ must employ to exhibit the observed route diversity. In particular, if we infer diversity sets by means of Property 1 and then search for the biggest diversity set associated with AS $X$ across prefixes, we actually

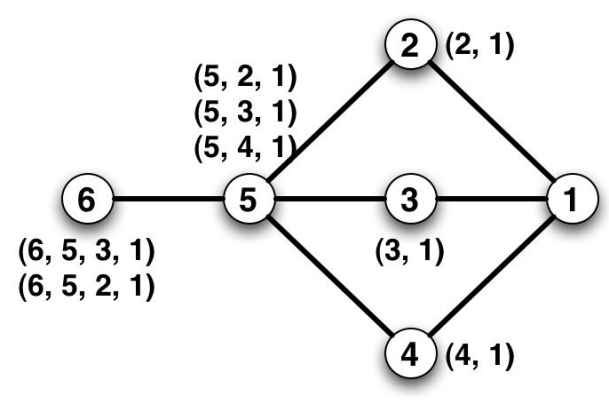

Figure 1. Example of network topology. Each node represents an AS and each edge one (or more) peering(s) between a pair of ASes. AS 1 origins prefix $p$. Each node is associated with a set of paths it uses to reach $p$ at time $t$.

infer a lower bound on the number of BGP routers in $X$. We denote the cardinality of the biggest diversity set associated with $X$ as its $\max -r d$. More formally, a lower bound for the BGP routers of $X$ is max-rd $(X)=\max _{S_{X}: \neq_{p, t} S_{X}}\left|S_{X}\right|$.

Consider the example in Figure. 1. AS 5 uses three distinct paths to reach AS 1 , namely $\pi_{1}=(5,2,1), \pi_{2}=$ $(5,3,1), \pi_{3}=(5,4,1)$. Hence, by Property 1 , the relation $\neq_{p, t}\left\{5_{2}, 5_{3}, 5_{4}\right\}$ holds and implies that AS 5 has at least 3 BGP routers. AS 6 uses two distinct paths to reach AS 1, both of which have AS 5 as a common neighbor. Hence, Property 1 implies that $6_{5}^{1} \neq_{p, t} 6_{5}^{2}$.

\section{Our Dataset}

The analyses shown throughout this paper rely on BGP data (both BGP RIBs and updates) collected by 574 CPs of the RIS [1] and RV [2] projects from 07/17/2007 to 07/30/2007 (called hereafter reference period).

Our dataset contains 538,341,994 updates (about 38M updates per day on average) with 12,351,221 distinct ASpaths on 76,014 distinct peerings and 26,491 distinct ASes. The number of prefixes is 293,154 .

For comparison, the dataset used in $[8,9]$ consists of a single snapshot of RIBs collected on 11/13/2005 from more than 1,300 observation points and contains 4,730,222 distinct AS-paths on 58,903 distinct peerings. Note that, even if the number of observation points is more than twice than ours, our dataset contains many more peerings and ASpaths. Hence, since route diversity can be inferred by comparing distinct paths collected at different vantage points (see Property 1), more AS-paths can result in the inference of more route diversity. We highlight that, while the two datasets refer to different time periods, we ascribe the difference in terms of number of distinct AS-paths mostly to 
the contribution of BGP updates. This intuition is backed by $[4,10]$, which show that taking BGP dynamics into account systematically results in more topology information than relying only on a static snapshot of the Internet.

\section{Extracting Diversity Relationships from a Dynamic Dataset}

How to extract diversity relationships from a static snapshot of the network has been already discussed in [8]. We move one step further by dealing with the problem of extracting $\neq_{p, t}$ relationships in a dynamic setting, taking into account routing changes. One of our goals is to evaluate how BGP dynamics affects the number of diversity relationships that can be inferred from data collected by multiple remote vantage points.

Our approach is as follows. We scan in chronological order the stream of BGP updates collected in a given time interval by all the CPs and we keep track of which paths are used by which AS over time. Namely, for every AS $X$ and prefix $p$ we keep in memory the used paths set ups $(X, p, t)$, i.e., the set of paths that are used by $X$ to reach prefix $p$ at time $t$, as observed by at least one CP. We also associate to every ups $(X, p, t)$ the number of CPs which observe prefix $p$ through AS $X$ at time $t$. By using a reference-counting like mechanism, we update ups by removing a path $\pi$ as soon as no CP sees AS $X$ reaching $p$ through $\pi$ anymore. Finally, we infer $\neq_{p, t}$ relationships associated with AS $X$ by applying Property 1 to the paths in $u p s(X, p, t)$.

Our implementation computes diversity sets out of a stream of BGP updates in near real-time. Namely, it took us less than 12 hours to run this computation on our whole reference period, using on average $2.4 \mathrm{~GB}$ memory on a common machine (2x Intel Xeon 2.80GHz CPUs, 4GB RAM).

It is important to note that BGP prefix aggregation can mislead our approach. In fact, the AS-path carried in an aggregated announcement does not identify a unique route to the destination prefix [7]. Thus, we deliberately disregard such announcements. We argue that this is a negligible limitation, since BGP prefix aggregation is renowned to be seldom performed. E.g., in our dataset, a RIB contains on average less than 100 aggregated announcements $(<0.05 \%)$.

When extracting diversity sets from BGP updates, a major concern is handling transient periods, because it could produce false positives. Consider two collector peers $c p_{1}$ and $c p_{2}$, both having a route $(X Y Z)$ to prefix $p$ at time $t_{0}$. Assume that the peering between $X$ and $Y$ fails at time $t_{1}\left(t_{1}>t_{0}\right)$, and $c p_{1}$ switches to an alternative path, say ( $X W Z$ ) at time $t_{2}\left(t_{2}>t_{1}\right)$. Due to the propagation delay of BGP messages, $c p_{2}$ could still be using path $(X Y Z)$ at time $t_{2}$. As a consequence, Property 1 applies; hence our algorithm would incorrectly infer $X_{Y} \neq_{p, t} X_{W}$. Ob-

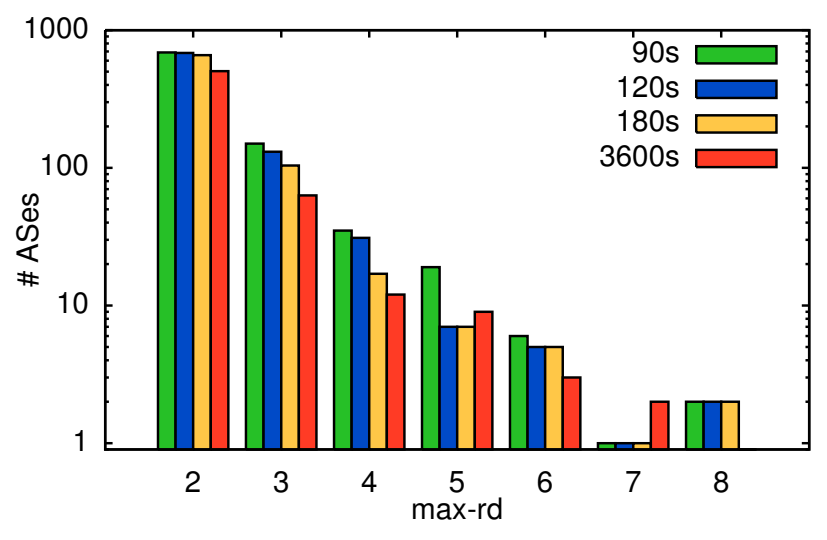

Figure 2. max-rd computed over the reference period using different values of the convergence time $\Delta$. ASes with max-rd $=1$ are omitted.

serve that BGP path exploration - i.e. discarding alternative paths that are gradually withdrawn (see, e.g., [6]) - additionally exacerbates this problem. We face such a shortcoming by defining a convergence time $\Delta$. Namely, when computing the used paths set, any prefix that underwent a routing change in the last $\Delta$ seconds is not considered for the inference of diversity relationships.

Different choices of $\Delta$ can lead to different diversity sets, potentially affecting the value of max-rd for a number of ASes. Namely, choosing a $\Delta$ smaller than the time it takes to update all the collector peers about a given event might result in false-positives. On the other hand, using bigger values of $\Delta$, we may disregard paths which actually represent diverse routes, producing false-negatives.

In order to assess this sensitivity, we ran our algorithm on our dataset with different values of $\Delta$. Fig. 2 shows the distribution of max-rd for $\Delta=90,120$, and 180 seconds. The distributions are almost unaffected by the specific choice of $\Delta$. As a further proof, we plot the distribution obtained by setting $\Delta=3600$ seconds, which is an extremely conservative convergence time. Observe that the values of max-rd are still quite comparable with the ones obtained with realistic values of $\Delta$. Backed from the results in [5], throughout this paper we will assume $\Delta=180$ seconds.

\section{Understanding Route Diversity in the In- ternet}

In this section we will characterize the route diversity phenomenon with respect to the metrics defined in Section 2 applied to our dataset. 


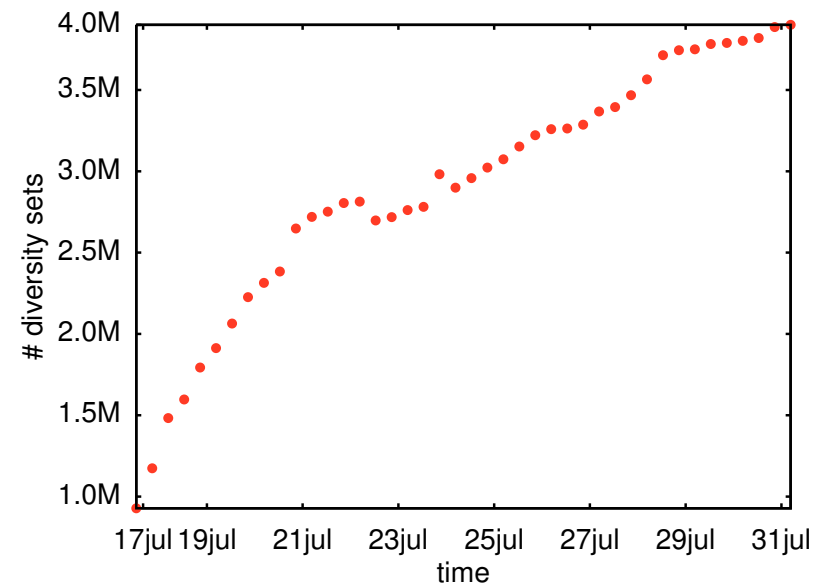

Figure 3. Cumulative number of diversity sets over time.

\subsection{The Impact of BGP Dynamics on Route Diversity}

As discussed in Section 4, our methodology extends [8] by accounting for the BGP dynamics. We will show the effect of extracting route diversity from BGP updates.

Figure 3 plots the cumulative number of distinct diversity sets inferred over time. In particular, the first data point represents the number of diversity sets extracted from the initial RIBs, while the data point at time $t$ represents the number of diversity sets extracted using both the initial RIBs and the BGP updates collected up to $t$. This number significantly increases over time. In particular, at the end of the reference period, we infer approximately four times the diversity sets obtained from the initial RIB snapshot. Note that the growth exhibits a pseudo-logarithmic trend, i.e., taking BGP dynamics into account can greatly influence the ability to capture route diversity only on relatively short time scales. On the other hand, considering larger time scales can potentially mislead the inference, because of changes in the network topology

Moreover, accounting for BGP dynamics allows us to identify a higher number of ASes exhibiting max-rd $>1$. Namely, we detected 795 such ASes over the whole reference period, while only 178 from the initial snapshot. Breaking down such ASes according to their values of max-rd, Figure 4 shows a significant improvement in the distribution of max-rd at the end of the reference period, compared to the values computed on the initial RIBs.

Overall, our results show that BGP dynamics play an important role in detecting the diversity relationships that can be observed by remote vantage points, hence our methodology can help build topology models where route diversity is

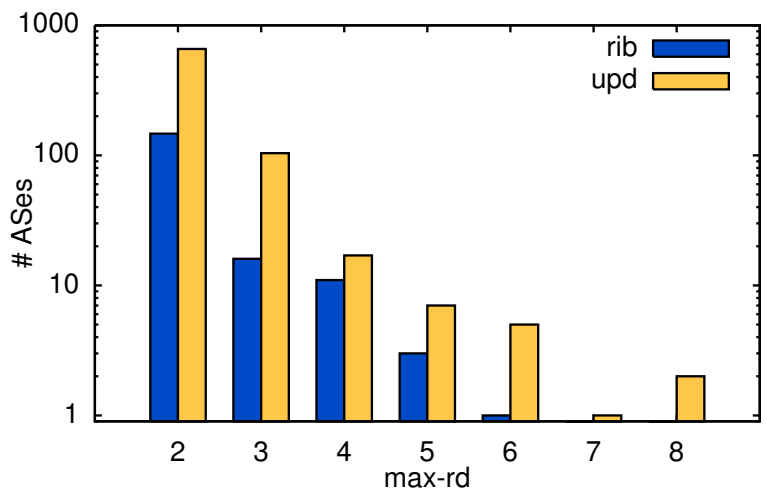

Figure 4. max-rd extracted from a RIB snapshot at the beginning of the reference period and from all the BGP updates collected during the reference period. ASes with max-rd $=$ 1 are omitted.

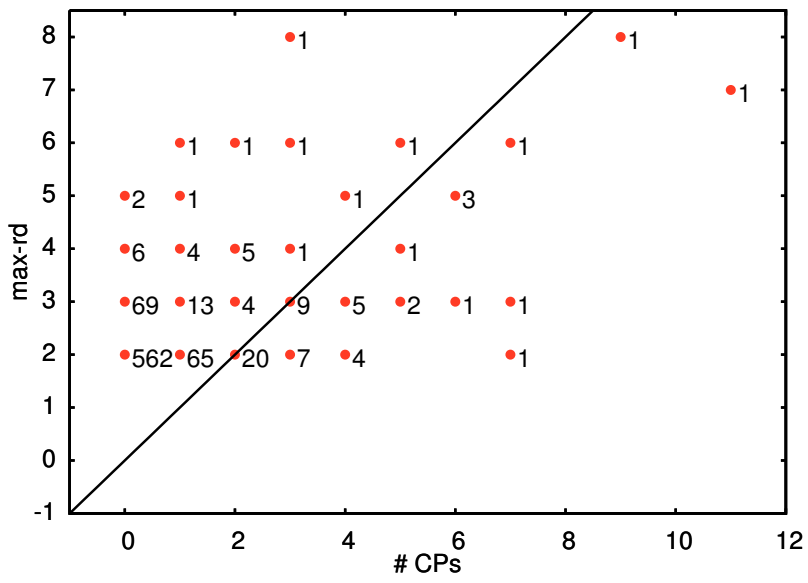

Figure 5. Each point $(x, y)$ is labelled with the number of ASes that host $x$ CPs and have max-rd $=y \cdot y=x$ line is also plotted for convenience.

captured more accurately.

\subsection{Sensitivity to Our Dataset}

In order to assess the scope of our results, we aim at understanding up to what extent the diversity relationships we extracted depend on our dataset.

Since max-rd relies on the availability of distinct paths as observed by any CPs, we analyze whether its value can be biased towards those ASes that host a large number of collector peers. Figure 5 plots the relationship between number of CPs hosted by an AS and its max-rd. It shows that most 


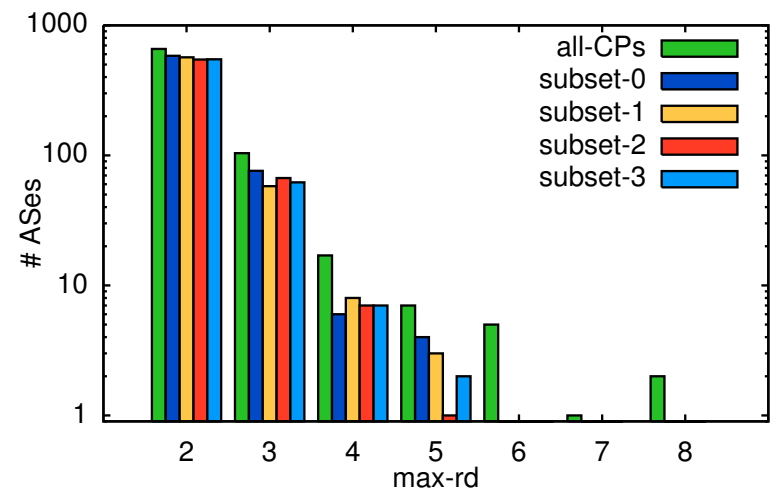

Figure 6. max-rd extracted from subsets of CPs. ASes with max-rd $=1$ are omitted.

of the diversity relationships are inferred for ASes hosting a few (or no) CPs, while a small number of ASes shows very low values of max-rd, although they host several CPs.

Moreover, the presence of a CP could influence not only the max-rd of the AS that hosts it, but also of others. To verify if this happens, we randomly partitioned the whole set of CPs into four sub-sets and we computed the max-rd over each of them. Figure 6 shows that max-rd distributions of all the sets are quite similar and closely resemble the max-rd distribution over all the CPs.

Overall, the max-rd metric seems to be only minimally affected by the number and the location of collector peers. As further comparison, we also note that the max-rd distribution at the beginning of the reference period (Figure 4) closely follows what presented in [8], both in trend and in values, even if the data sources greatly differ both in the time period and in the number and location of the used vantage points.

Unfortunately, this property does not hold for the number of prefixes exhibiting route diversity. (Note that, overall, more than 230k prefixes in our dataset exhibit some route diversity - about $78 \%$ of all routed prefixes.) Figure 7 plots the relationship between the ASes hosting no CP and exhibiting some route diversity and the prefixes the diversity is observed on. Note that the distribution clearly exhibits a power-law trend. By contrast, the relationship between the ASes hosting CPs and exhibiting some route diversity and the prefixes the diversity is observed on (Figure 8) features an irregular distribution. Hence, we suspect that the number of prefixes for which some route diversity can be observed in a given AS is a metric that can be heavily biased by the choice of vantage points.

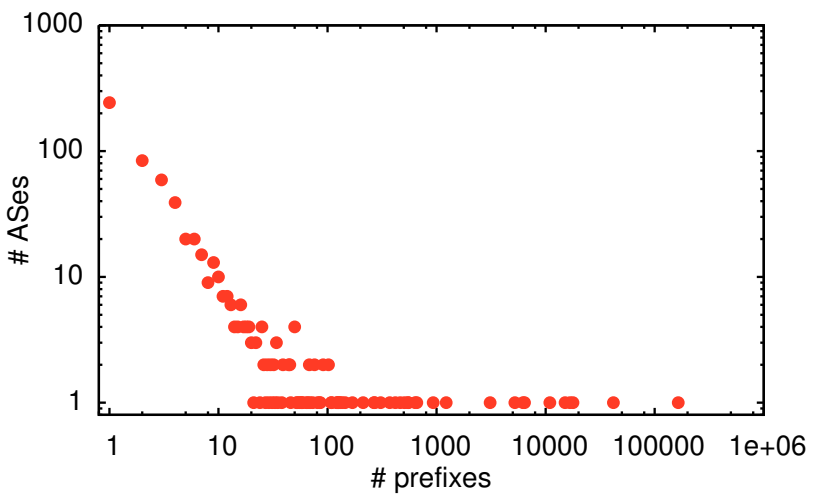

Figure 7. Each point $(x, y)$ represents $y$ ASes that host no CP and exhibit max-rd $>1$ on $x$ prefixes.

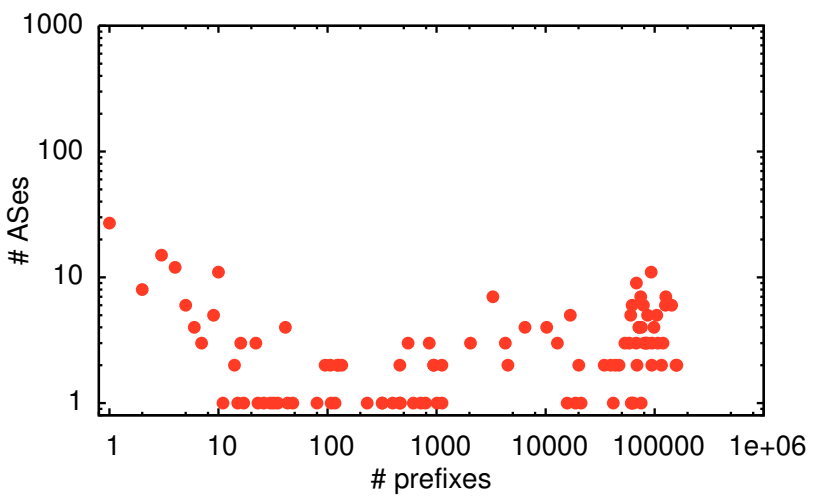

Figure 8. Each point $(x, y)$ represents $y$ ASes that host at least one CP and exhibit max-rd > 1 on $x$ prefixes.

\subsection{Relating Route Diversity to the Inter- net Hierarchy}

We now investigate which types of ASes exhibit route diversity that can be observed by remote vantage points. Our intuition is that ASes with a large number of customers most likely maintain multiple routes toward the same destination, in order to ensure reliable connectivity. To estimate the number of customers, we relied on data provided by CAIDA [3]. Namely, CAIDA publishes a ranking of the ASes based on the cardinality of their customer cones, estimated as described in [11]. Basically, the topmost ASes in such a ranking are supposed to have the largest number of customers in the Internet.

We thus compared the max-rd with CAIDA's ranking related to the same reference period. Figure 9 shows that ASes with high values of max-rd usually have lots of cus- 


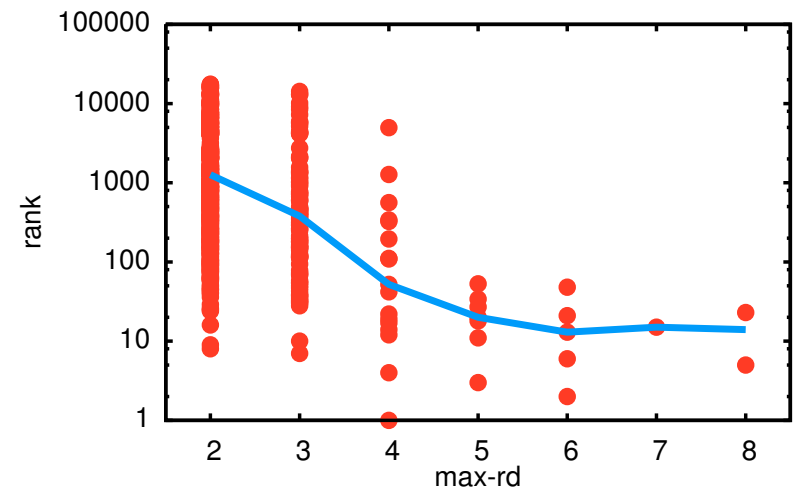

Figure 9. Each point $(x, y)$ represents an AS having max-rd $=x$ and CAIDA's rank $=y$. The solid line shows the median value of rank among ASes having the same value of max-rd.

tomers and, on the other hand, ASes with low values of max-rd generally have a few customers. Figure 10 further stresses the difference of max-rd distribution of ASes belonging to different portions of the ranking (namely, top-n ASes, with $n \in\{10,50,100\}$ ).

Our analysis shows that the location of ASes in the Internet hierarchy strongly impacts the diversity that can be observed by remote vantage points.

\section{Conclusions and Future Work}

In this paper we studied the characteristics of the BGP route diversity that can be observed by passive remote vantage points. We defined a methodology to extract diversity relationships over time from a continuous stream of BGP updates. By accounting for the routing dynamics, we inferred four times more diversity relationships than only using a static snapshot of the Internet routing configuration. We also showed that the behavior of the max-rd metric is unlikely to be biased from the specific choice of the dataset. Finally, we correlated the observed route diversity of ASes to their position in the Internet hierarchy.

As future work, we plan to exploit correlations and overlapping among diversity sets associated with different prefixes in order to improve our ability in extracting diversity relationships. We also want to perform a historical analysis of both max-rd values and diversity sets, in order to better understand their behavior over time. In general, we believe that being aware of which portion of the internal AS structure can be observed from remote vantage points is an important research effort towards a better understanding of the Internet routing.

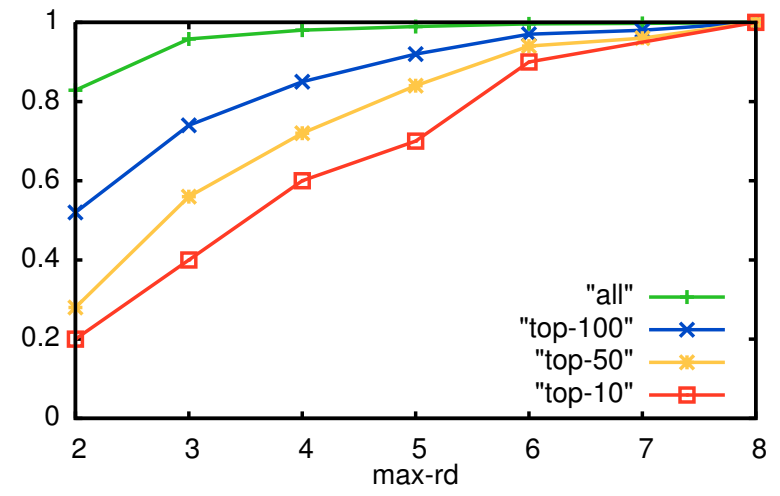

Figure 10. CDF of max-rd for the top $n$ ASes, $n \in\{10,50,100\}$. all refers to the whole topology.

\section{References}

[1] http://www.ripe.net/ris/.

[2] http://www.routeviews.org/.

[3] http://as-rank.caida.org/.

[4] B. Zhang, R. Liu, D. Massey, and L. Zhang. Collecting the Internet AS-Level Topology. ACM SIGCOMM CCR, 2005.

[5] C. Labovitz, et al. The Impact of Internet Policy and Topology on Delayed Routing Convergence. In IEEE INFOCOM, 2001.

[6] R. Oliveira, B. Zhang, D. Pei, R. Izhak-Ratzin and L. Zhang. Quantifying Path Exploration in the Internet. In $I M C, 2006$.

[7] Y. Rekhter, T. Li, and S. Hares. A Border Gateway Protocol 4 (BGP-4). IETF RFC 4271, 2006.

[8] W. Muhlbauer, A. Feldmann, O. Maennel, M. Roughan and S. Uhlig. Building an AS-Topology Model that Captures Route Diversity. In ACM SIGCOMM, 2006.

[9] W. Mühlbauer, S. Uhlig, B. Fu, M. Meulle and O. Maennel. In Search for an Appropriate Granularity to Model Routing Policies. In ACM SIGCOMM, 2007.

[10] X. A. Dimitropoulos, D. V. Krioukov and G.F. Riley. Revisiting Internet AS-Level Topology Discovery. In PAM, 2005.

[11] X. Dimitropoulos, et al. AS Relationships: Inference and Validation. ACM SIGCOMM CCR, 2007. 